\title{
Holonomic Character and Local Monodromy Structure of Feynman Integrals
}

\author{
Masaki Kashiwara* \\ Mathematical Institute, Nagoya University, Nagoya, Japan, and \\ Department of Mathematics, Harvard University, Cambridge, Massachusetts, USA \\ Takahiro Kawai ${ }^{\star \star}$ \\ Research Institute for Mathematical Sciences, Kyoto University, Kyoto, Japan, and \\ Department of Mathematics, University of California, Berkeley, California, USA
}

\begin{abstract}
We prove that the micro-local holonomic structure controls the local monodromy structure of functions involved. This result plays an essential role in investigating "hierarchical principle" in perturbation theory.
\end{abstract}

\section{§1. Introduction}

At the occasion of Kyoto symposium $(M \cap \Phi)$ in 1975 , Sato [15] emphasized the importance of holonomic systems of micro-differential equations ${ }^{1}$ in investigating the $S$-matrix and related functions. At least in the case of Feynman integrals this point of view (i.e. the use of over-determined system of linear differential equations) was also emphasized by Regge [12] as early as 1967 .

In this approach, the first thing to do is to establish the fact that the $S$-matrix and/or Feynman integrals satisfy some holonomic systems of (micro-)differential equations. Partial results were given for Feynman integrals by Barucchi and Ponzaro [1] and Sato [15] and for the $S$-matrix by Kawai and Stapp [5, 6]. In this direction a decisive result has recently been given for arbitrary Feynman integrals by Kashiwara and Kawai [3,4].

Having this situation in mind, we show in this article how the holonomic structure controls the local sheet structure of Feynman integrals. More precisely, we show in Theorem 2 that our main result (Theorem 1) applied to Feynman integrals entails that the local monodromy structure of the Feynman integral associated with a Feynman diagram $D$ controls that of the Feynman integral associated with the "daughter" diagram $D^{\prime}$ of $D$ under moderate conditions. Thus our results find an intimate connection with the celebrated "hierarchical principle" proposed by

$\star$ Supported by National Science Foundation

$\star \star$ Supported by Miller Institute for Basic Research in Science

1 In Sato et al. [16], a holonomic system is called a maximally overdetermined system and a microdifferential equation (operator, resp.) is called a pseudo-differential equation (operator, resp.). Here we change our terminology according to the suggestion of Prof. Sato. We also use the terminology "holonomicity of a function" to indicate the holonomic character of the function, i.e., the fact that the function satisfies a holonomic system of (micro-)differential equations. 
Cambridge group (see e.g. Landshoff et al. [7]). In our formulation, "hierarchy" should be assigned through the existence of a micro-differential operator $Q$ discovered by the authors. (See p. 22 of Sato [15]. Note that Regge [12] has also conjectured the existence of an operator essentially the same as $Q$.) This will be one of the most important steps when we try to achieve the charming program of Regge for the systematic study of Feynman integrals. See Pham [9], Regge [12, 13], Speer and Westwater [18], Ponzano et al. [10,11], Regge et al. [14], and references cited there for related topics.

\section{§ 2. Micro-local Holonomic Character and Local Monodromy Structure}

We begin our discussions by showing some lemmas on the analytic properties of microfunction solutions of a holonomic system of micro-differential equations. In applying our main theorem to the investigation of Feynman integrals, we can bypass the use of these lemmas by using our basic result (Kashiwara and Kawai $[3,4]$ ) on the existence of a holonomic system of linear differential equations that a Feynman integrals should satisfy. However, in order to embody the fascinating idea of Sato [15], i.e. the micro-local study of Feynman integrals and the $S$-matrix, these lemmas (especially Lemma 3 ) are crucial in the sense that purely micro-local information can control the behavior of the solutions involved in complex domain.

Before stating our results, we prepare some notations.

Let $M$ be a real analytic manifold and let $X$ be its complexification. Let $x_{0}^{*}$ be a point in $\sqrt{-1} S^{*} M$. We will choose a local coordinate system $\left(t, x_{1}, \ldots, x_{n}\right)$ so that $x_{0}^{*}=(0,0 ; \sqrt{-1} d t \infty)$. We denote by $F$ the projection from $X$ to $\mathbb{C}^{n}$ defined by $(t, x) \mapsto x$. Accordingly a point in $P^{*} X$ will be denoted by $(t, x ; \tau, \xi)$ and a point in $\sqrt{-1} S^{*} M$ will be denoted by $(t, x ; \sqrt{-1}(\tau, \xi))$.

Let $\gamma$ be a closed loop in $X-Y$ for a hypersurface $Y$ in $X$ and $f(t, x)$ be a multivalued analytic function defined on $X-Y$ (i.e. holomorphic on the universal covering space $\overparen{X-Y}$ of $X-Y$ ). Then we denote by $T_{\gamma} f$ the function obtained by analytically continuing $f(t, x)$ along $\gamma$ starting from a point on a specified sheet.

In order to simplify our notations, we shall use the following conventional notations in the sequel.

(1) The point $(t, x)=(0,0)$ is denoted by 0 .

(2) The projection from $P^{*} X\left(\sqrt{-1} S^{*} M\right.$, resp.) to $X(M$, resp. $)$ is denoted by $\pi$.

(3) For a topological space $Z, \tilde{Z}$ denotes its universal covering space.

(4) $B(\varepsilon)=\left\{x \in \mathbb{C}^{n} ;|x|<\varepsilon\right\}$.

(5) $B\left(x_{0}, \varepsilon\right)=\left\{x \in \mathbb{C}^{n} ;\left|x-x_{0}\right|<\varepsilon\right\}$ for $x_{0} \in \mathbb{C}^{n}$

(6) $D(\delta)=\{t \in \mathbb{C} ;|t|<\delta\}$.

(7) $D\left(t_{0}, \delta\right)=\left\{t \in \mathbb{C} ;\left|t-t_{0}\right|<\delta\right\} \quad$ for $t_{0} \in \mathbb{C}$. 
Lemma 1. Let $V$ be a Lagrangian variety of $P^{*} X$. Assume that

(8) $V \cap \pi^{-1}(0)=\{d t \infty\}$

holds. Then, for any positive integer $\varepsilon$, there exists a neighborhood $U$ of 0 such that (9) $\{(t, x) \in U ;|t|>\varepsilon|x|\} \subset X-\pi(V)$.

Proof. If the conclusion were false, we could find $\varepsilon>0$ and a real analytic curve $(t(\lambda)$, $x(\lambda))_{0 \leqq \lambda \leqq 1}$ in $Y$ which satisfies the following conditions:

(10) $|t(\lambda)| \geqq \varepsilon|x(\lambda)|$,

(11) $(t(0), x(0))=0$.

Since $\left.\pi\right|_{V}: V \rightarrow Y$ is a finite surjective map, we can lift up this curve on $V$. We denote the corresponding point on $V \subset P^{*} X$ by $(t(\mu), x(\mu) ; p(\mu))$. Here $p$ is by definition $-\xi / \tau$, the inhomogeneous coordinate in a neighborhood of $d t \infty$ in $P^{*} X$. Note that condition (8) implies that $p(0)=0$. Since $V$ is Lagrangian, the canonical 1 -form $\omega$ $=d t-\langle p, d x\rangle$ restricted to $V$ is zero by the definition. Therefore

(12) $\quad d t(\mu) / d \mu=\langle p(\mu), d x(\mu) / d \mu\rangle$

holds along the curve $(t(\mu), x(\mu) ; p(\mu))_{0 \leqq \mu \leqq 1} \subset V$. On the other hand, conditions (10) and (11) imply that

(13) $\left|t^{\prime}(0)\right| \geqq \varepsilon\left|x^{\prime}(0)\right|$.

Since $p(\mu)$ is analytic in $\mu$ and $p(0)=0$, this contradicts (12).

This completes the proof of Lemma 1.

In the situation discussed by Lemma 1, we may assume that $V$ is closed in the inverse image by $\pi$ of a small neighborhood $U=D\left(\delta_{0}\right) \times B\left(\varepsilon_{0}\right)$ of 0 and that $V \cap \pi^{-1}(U) \rightarrow U$ is a finite map. Then

(14) $Y=\pi(V) \cap U$ is a closed hypersurface of $U=D\left(\delta_{0}\right) \times B\left(\varepsilon_{0}\right)$.

Choosing $\varepsilon_{0}$ sufficiently small, we may further assume

$$
F^{-1}\left(B\left(\varepsilon_{0}\right)\right) \cap Y \rightarrow B\left(\varepsilon_{0}\right) \text { is a finite map. }
$$

Now define $H$ as the image of the points of $Y$ where $Y \rightarrow B\left(\varepsilon_{0}\right)$ is not locally isomorphic. Then

$$
F^{-1}\left(B\left(\varepsilon_{0}\right)-H\right) \cap(U-Y) \rightarrow B\left(\varepsilon_{0}\right)-H
$$

is locally topologically trivial. Further we may choose $\delta_{0}$ and $\varepsilon_{0}$ so that

(16) $\varepsilon_{0}<\delta_{0}$

and

$$
\left\{(t, x) \in D\left(\delta_{0}\right) \times B\left(\varepsilon_{0}\right) ;|t|>|x|\right\} \subset X-Y
$$

hold. 
Then we have the following

Lemma 2. Assume that conditions (14)-(17) are fulfilled. Then for any $x$ in $B\left(\varepsilon_{0}\right)-H$, the canonical map

$$
\pi_{1}\left(F^{-1}(x) \cap\left(D\left(\delta_{0}\right) \times B\left(\varepsilon_{0}\right)-Y\right)\right) \rightarrow \pi_{1}\left(D\left(\delta_{0}\right) \times B\left(\varepsilon_{0}\right)-Y\right)
$$

is surjective for sufficiently small $\varepsilon_{0}$.

Proof. First recall the following well-known exact sequence due to HurewiczSteenrod:

$$
\left.\pi_{1}\left(D\left(\delta_{0}\right) \times B\left(\varepsilon_{0}\right)-Y\right) \cap F^{-1}(x)\right) \stackrel{i}{\rightarrow} \pi_{1}\left(D\left(\delta_{0}\right) \times B\left(\varepsilon_{0}\right)-Y-F^{-1}(H)\right) \stackrel{j}{\rightarrow} \pi_{1}\left(B\left(\varepsilon_{0}\right)\right.
$$

$-H)$.

Since $F^{-1}(H)$ is of real codimension 2 in $X$, the map $k$ from $\pi_{1}\left(D\left(\delta_{0}\right) \times B\left(\varepsilon_{0}\right)-Y\right.$ $\left.-F^{-1}(H)\right)$ to $\pi_{1}\left(D\left(\delta_{0}\right) \times B\left(\varepsilon_{0}\right)-Y\right)$ is surjective.

We shall now show that

$$
l=k \circ i: \pi_{1}\left(\left(D\left(\delta_{0}\right) \times B\left(\varepsilon_{0}\right)-Y\right) \cap F^{-1}(x)\right) \rightarrow \pi_{1}\left(D\left(\delta_{0}\right) \times B\left(\varepsilon_{0}\right)-Y\right)
$$

is surjective. For this purpose, it suffices to show that for any $\gamma$ in $\pi_{1}\left(B\left(\varepsilon_{0}\right)-H\right)$ we can find $\gamma^{\prime} \in \pi_{1}\left(D\left(\delta_{0}\right) \times B\left(\varepsilon_{0}\right)-Y-F^{-1}(H)\right)$ so that $j\left(\gamma^{\prime}\right)=\gamma$ and $k\left(\gamma^{\prime}\right)=1$ hold, because $k$ is surjective. By conditions (16) and (17), we may define $\gamma^{\prime}$ by $\{t \in \mathbb{C} ; t$ $\left.=t_{0}\right\} \times \gamma$ with $\delta_{0}>\left|t_{0}\right|>\varepsilon_{0}$. It is clear that $\gamma^{\prime}$ is trivial in $\pi_{1}\left(\left\{(t, x) \in D\left(\delta_{0}\right) \times B\left(\varepsilon_{0}\right) ;|t|\right.\right.$ $>|x|\})$, and hence also trivial in $\pi_{1}\left(D\left(\delta_{0}\right) \times B\left(\varepsilon_{0}\right)-Y\right)$.

After these preparations, we obtain the following

Lemma 3. Let $f(t, x)$ be a hyperfunction on $M$ which satisfies following conditions:

$$
\text { S.S. } f \cap \pi^{-1}(0)=\{\sqrt{-1} d t \infty\}
$$

(21) $s p(f(t, x))^{2}$ satisfies a holonomic system $\mathfrak{M}=\mathscr{E} / \mathscr{J}^{3}$ of micro-differential equations.

(22) The characteristic variety $V$ of $\mathfrak{M}$ satisfies condition (8).

Let $\tilde{f}(t, x)$ be a holomorphic function whose boundary value $\alpha(\tilde{f})^{2}$ is $f$. (Existence of $\tilde{f}$ is guaranteed by condition (20).) Then $\tilde{f}(t, x)$ can be analytically continued so that it is multi-valued analytic on $U-Y=U-\pi(V)$ for sufficiently small neighborhood $U$ of 0 .

Proof. First note that condition (20) implies that $\tilde{f}(t, x)$ is holomorphic in $\{(t$, $\left.x) \in D\left(\delta_{0}\right) \times B\left(\varepsilon_{0}\right) ; \operatorname{Im} t>|\operatorname{Im} x|\right\}$ for sufficiently small $\delta_{0}, \varepsilon_{0}>0$. Let $P_{j}\left(t, x, D_{t}, D_{x}\right)(j$ $=1, \ldots, N)$ be micro-differential operators which generates the ideal $\mathscr{J}$. By Weierstrass type theorem for micro-differential operators (Sato et al. [16], Chapter II, §2.2), we may assume without loss of generality that $P_{j}$ is a differential operator in $D_{x}$. We may further assume that order $P_{j} \leqq-1$. Then we can find a linear differential operator $A_{j}\left(t, t^{\prime}, x, D_{x}\right)$ in $D_{x}$ with holomorphic coefficients in $\left(t, t^{\prime}, x\right)$ near the orgin such that

$$
\operatorname{sp}\left(\alpha\left(\int_{c}^{t} A_{j}\left(t, t^{\prime}, x, D_{x}\right) \tilde{f}\left(t^{\prime}, x\right) d t^{\prime}\right)\right)=P_{j}\left(t, x, D_{t}, D_{x}\right) \operatorname{sp}(\alpha(\tilde{f}))
$$

2 As for the definition of $\alpha(\tilde{f})$ and $s p(f)$, we refer to $\S 1.5$ of Sato et al. [16], Chapter I

$3 \mathscr{E}$ denotes the sheaf of micro-differential operators of finite order 
holds for sufficiently small $c$ (see Kashiwara and Kawai [2], §3). Hence we may assume that $A_{j}\left(t, t^{\prime}, x, D_{x}\right)$ is defined for $\left(t, t^{\prime}, x\right) \in D\left(\delta_{0}\right) \times D\left(\delta_{0}\right) \times B\left(\varepsilon_{0}\right), c \in D\left(\varepsilon_{0}\right)$ with $\operatorname{Im} c>0$ and that $\int_{c}^{t} A_{j}\left(t, t^{\prime}, x, D_{x}\right) \tilde{f}\left(t^{\prime}, x\right) d t^{\prime}$ is defined and holomorphic if $(t$, $x) \in D\left(\delta_{1}\right) \times B\left(\varepsilon_{0}\right)$ for $\delta_{0} \gg \delta_{1}>0$. Furthermore we may assume that $\varepsilon_{0}$ and $\delta_{0}$ satisfy conditions (14)-(17) so that Lemma 2 is applicable. We shall take $\varepsilon_{1}$ such that $0<\varepsilon_{1}$ $\leqq \varepsilon_{0}, \delta_{1}$.

We now embark on the proof of the extensibility of $\tilde{f}(t, x)$. Fix a point $x_{0}$ in $B\left(\varepsilon_{1}\right)$ $-H$ and define $W=B\left(x_{0} ; \varrho\right) \subset B\left(\varepsilon_{1}\right)-H$ for sufficiently small $\varrho>0$. Note that $Y \cap D\left(\delta_{1}\right) \times B\left(\varepsilon_{1}\right) \cap F^{-1}(x)$ is given by $\left\{a_{1}(x), \ldots, a_{m}(x)\right\}$ by holomorphic function $a_{j}(x)$ in $x \in W$. We next define $G=D\left(\delta_{1} ; x_{0}, \sigma\right)$ by $D\left(\delta_{1}\right)-\left\{t \in \mathbb{C} ;\left|t-a_{j}\left(x_{0}\right)\right| \leqq \sigma, j\right.$ $=1, \ldots, m\}$ for a sufficiently small $\sigma$ and denote its universal covering space by $\tilde{G}$. We denote by $\varpi$ the projection from $\tilde{G}$ to $G$.

We shall prove that $\tilde{f}(t, x)$ can be analytically continued all over $\tilde{G} \times W$. First define $\Omega$ as a maximal connected subset of $\tilde{G}$ that satisfies the following property. (24) $\tilde{f}(t, x)$ is analytically continued all over $\Omega \times W$.

We want to show $\Omega=\tilde{G}$. For this purpose we define an auxiliary set $\Omega^{\prime}$ as follows:

(25) $\Omega^{\prime}$ is a set of point $p$ in $\Omega$ such that $\int_{t_{0}}^{t} A_{j}\left(t, t^{\prime}, x, D_{x}\right) \tilde{f}\left(\varpi_{U}^{-1}\left(t^{\prime}\right), x\right) d t^{\prime}(j=1, \ldots, N)$ is holomorphic if $t \in D\left(\delta_{1}\right)$ and $x \in W$. Here $U$ is an open neighborhood of $p$ such that $U \rightarrow \varpi(U)$ is isomorphic and we denote by $\varpi_{U}$ the restriction of $\varpi$ to $U$, and $t_{0}=\varpi(p)$.

Now we shall show $\Omega^{\prime}=\Omega$. We first show $\Omega^{\prime} \neq \emptyset$. By choosing $c^{\prime} \in D\left(\delta_{1}\right)$ with $\operatorname{Im} c^{\prime}$ $>\varepsilon_{1}, \int_{c}^{c^{\prime}} A_{j}\left(t, t^{\prime}, x, D_{x}\right) \tilde{f}\left(t^{\prime}, x\right) d t^{\prime}$ is holomorphic if $(t, x) \in D\left(\delta_{1}\right) \times B\left(\varepsilon_{1}\right)$, because $A_{j}\left(t, t^{\prime}\right.$, $\left.x, D_{x}\right)^{c}$ and $\tilde{f}\left(t^{\prime}, x\right)$ are holomorphic when $t \in D\left(\delta_{1}\right), x \in B\left(\varepsilon_{1}\right)$ and $t^{\prime} \in D_{c^{\prime}}\left(\delta_{0}\right)$, $\operatorname{Im} t^{\prime}>\varepsilon_{1}$. Therefore $\int_{c^{\prime}}^{t} A_{j}\left(t, t^{\prime}, x, D_{x}\right) \tilde{f}\left(t^{\prime}, x\right) d t^{\prime}=\int_{c}^{t} A_{j}\left(t, t^{\prime}, x, D_{x}\right) \tilde{f}\left(t^{\prime}, x\right) d t^{\prime}-\int_{c}^{c^{\prime}} A_{j}\left(t, t^{\prime}, x, D_{x}\right)$ $\tilde{f}\left(t^{\prime}, x\right) d t^{\prime}$ is holomorphic for $(t, x) \in D^{c}\left(\delta_{1}\right) \times B\left(\varepsilon_{1}\right)$. This implies that $\Omega^{\prime} \neq \emptyset$.

Secondly we shall show that $\Omega^{\prime}$ is open. Let $p$ be a point in $\Omega^{\prime}$. Take $p^{\prime}$ in $U$ sufficiently close to $p$. Then $\int_{\varpi_{\left(p^{\prime}\right)}}^{t} A_{j}\left(t, t^{\prime}, x, D_{x}\right) \tilde{f}\left(\left(\varpi_{U}^{-1}\left(t^{\prime}\right), x\right) d t^{\prime}=\int_{\varpi\left(p^{\prime}\right)}^{t_{0}} A_{j}\left(t, t^{\prime}, x, D_{x}\right)\right.$ $f\left(\varpi_{U}^{-1}\left(t^{\prime}\right), x\right) d t^{\prime}+\int_{t_{0}}^{t} A_{j}\left(t, t^{\prime}, x, D_{x}\right) f\left(\varpi_{U}^{-1}\left(t^{\prime}\right), x\right) d t^{\prime}$ is clearly holomorphic for $(t$, $x) \in D\left(\delta_{1}\right) \times W$. Therefore $\Omega^{\prime}$ is open.

Lastly we shall show that $\Omega^{\prime}$ is closed in $\Omega$. Let $p_{0}$ be in $\bar{\Omega}^{\prime} \cap \Omega$. Choose $p_{1} \in \Omega^{\prime}$ sufficiently close to $p_{0}$ and define $t_{1}$ by $\varpi\left(p_{1}\right)$. Since $p_{1} \in \Omega^{\prime}, \int_{t_{1}}^{t} A_{j}\left(t, t^{\prime}, x, D_{x}\right)$ $f\left(\varpi_{U}^{-1}\left(t^{\prime}\right), x\right) d t^{\prime}$ is holomorphic for $(t, x) \in D\left(\delta_{1}\right) \times W$. Further, $\int_{t_{0}}^{t^{\prime}} A_{j}\left(t, t^{\prime}, x, D_{x}\right)$ $f\left(\varpi_{U}^{-1}\left(t^{\prime}\right), x\right) d t^{\prime}$ is holomorphic for $(t, x) \in D\left(\delta_{1}\right) \times W$, because $p_{0} \in \Omega$ and because the coefficients of $A_{j}$ 's are holomorphic in $\left(t, t^{\prime}, x\right) \in D\left(\delta_{1}\right) \times D\left(\delta_{1}\right) \times B\left(\varepsilon_{1}\right)$. Therefore $\int_{t_{0}}^{t} A_{j}\left(t, t^{\prime}, x, D_{x}\right) f\left(\varpi_{U}^{-1}\left(t^{\prime}\right), x\right) d t^{\prime}=\int_{t_{1}}^{t} A_{j}\left(t, t^{\prime}, x, D_{x}\right) f\left(\varpi_{U}^{-1}\left(t^{\prime}\right), x\right) d t^{\prime}+\int_{t_{0}}^{t_{1}} A_{j}\left(t, t^{\prime}, x, D_{x}\right)$ 
$f\left(\varpi_{U}^{-1}\left(t^{\prime}\right), x\right) d t^{\prime}$ is holomorphic for $(t, x) \in D\left(\delta_{1}\right) \times W$. This complete the proof of the claim that $\Omega=\Omega^{\prime}$.

Now we shall show that $\Omega=\tilde{G}$. Again, it is clear that $\Omega \neq \emptyset$ and $\Omega$ is open. Hence it suffices to show that $\Omega$ is closed. Let $p_{0}$ be a point in $\partial \Omega$. Choose a point $p_{1}$ sufficiently close to $p_{0}$ so that it belongs to $U$ chosen in (25). Define $t_{j}$ by $\varpi\left(p_{j}\right)(j=0$, $1)$. We may assume that

$$
\begin{aligned}
& \varpi(U) \supset D\left(t_{1}, \kappa\right), \\
& \left|t_{0}-t_{1}\right|<\kappa,
\end{aligned}
$$

and

$$
\left|t_{1}\right|<\delta_{1}-\kappa
$$

hold for some $\kappa>0$. Define $O_{c . x_{1}}$ by $\left\{(t, x) ;\left|t-t_{1}\right|^{2} / c+\left|x-x_{1}\right|^{2}<\left(\varrho^{2}-\left|x_{1}-x_{0}\right|^{2}\right) / 2\right\}$ for

$$
x_{1} \in W=B\left(x_{0}, \varrho\right) \text { and } 0<c<2 \kappa^{2} /\left(\varrho^{2}-\left|x_{0}-x_{1}\right|^{2}\right) .
$$

Clearly $\overline{O_{c, x_{1}}} \subset D\left(t_{1}, x\right) \times B\left(x_{0}, \varrho\right)$ and $O_{c_{1}, x_{1}} \subset O_{c_{2}, x_{1}}$ for $0<c_{1}<c_{2}$. We next define a real hypersurface $S_{c, x_{1}}$ by the boundary of $O_{c, x_{1}}$. It is clear that for any $x \in B\left(x_{0}, \varrho\right)$ we can find some $c<\kappa^{2} /\left(\varrho^{2}-\left|x_{0}-x_{1}\right|^{2}\right)$ so that $\left(t_{0}, x\right) \in S_{c, x_{1}}$. Therefore it suffices to show that

$$
f\left(\varpi_{U}^{-1}(t), x\right) \text { is holomorphic in } O_{c, x_{1}}
$$

implies

$$
\tilde{f}\left(\varpi_{U}^{-1}(t), x\right) \text { is holomorphic in } \overline{O_{c, x_{1}}} .
$$

On the other hand, the property (30) guarantees that it defines a well-defined microfunction $\mu$ on $S_{c, x_{1}}$ as its boundary value. Further $P_{j}\left(t, x, D_{x}\right) \mu$ is given by the boundary value of $\int_{t_{1}}^{t} A_{j}\left(t, t^{\prime}, x, D_{x}\right) \tilde{f}\left(\varpi_{U}^{-1}\left(t^{\prime}\right), x\right) d t^{\prime}$ as long as any complex hyperplane $\{(t, x) ;\langle x, \xi\rangle=a\}$ is not tangent to $S_{c, x_{1}}$ (Theorem 3.6 of Kashiwara and Kawai [2]). Note that $S_{c, x_{1}}$ is tangent to $\{(t, x) ;\langle x, \xi\rangle=a\}$ if and only if $t=t_{1}$. This fact guarantees us that we do not need to worry about such a point because $\tilde{f}\left(\varpi_{U}^{-1}(t), x\right)$ is holomorphic in a neighborhood of $\left\{t_{1}\right\} \times W$. Since $\int_{t_{1}}^{t} A_{j}\left(t, t^{\prime}, x, D_{x}\right)$ $\tilde{f}\left(\varpi_{U}^{-1}\left(t^{\prime}\right), x\right) d t^{\prime}$ is holomorphic for $(t, x) \in D\left(\delta_{1}\right) \times B\left(x_{0}, \varrho\right)$ due to the way of choosing $p_{1}$, it is holomorphic in a neighborhood of $\overline{O_{c, x_{1}}}$ for any $\left(c, x_{1}\right)$ that satisfies (29). In fact condition (28) guarantees that $D\left(t_{1}, \kappa\right) \subset D\left(\delta_{1}\right)$. Therefore (31) follows from the invertibility of elliptic micro-differential operators if $S_{c, x_{1}}$ is not characteristic with respect to $\mathfrak{M}$. This assertion of non-characteristicness is obvious, because $S_{c, x_{1}}$ is disjoint from $\pi(V)=Y$. Therefore $\Omega$ is closed. Thus we have verified that $\Omega=\tilde{G}$.

Let $\varpi_{1}$ be a projection from $\widetilde{D\left(\delta_{1}\right) \times B\left(\varepsilon_{1}\right)}-Y$ to $D\left(\delta_{1}\right) \times B\left(\varepsilon_{1}\right)-Y$. Then, for $x_{0} \in B\left(\varepsilon_{1}\right)-H, \varpi_{1}^{-1} F^{-1}\left(x_{0}\right)$ is a universal covering of $D\left(\delta_{1}\right)-\left\{a_{1}\left(x_{0}\right), \ldots, a_{m}\left(x_{0}\right)\right\}$ by Lemma 2 and hence the union of the images of $\tilde{G} \times W$ is a neighborhood of $\varpi_{1}^{-1} F^{-1}\left(x_{0}\right)$. Since this holds for any $x_{0}, \tilde{f}$ can be prolonged to a holomorphic function defined on $\varpi_{1}^{-1}\left(D\left(\delta_{1}\right) \times B\left(\varepsilon_{1}\right)-Y-F^{-1}(H)\right)$. 
So far we have discussed the extensibility of $\tilde{f}$ outside $H$. We finally show that $H$ has nothing to do with us.

Let $\varphi(t, x)$ be a defining function of the hypersurface $Y$. Note that $Y$ is a hypersurface because $V$ is of purely dimension $n$ and $V \rightarrow Y$ is a finite map.

We consider the map $\left(t^{\prime}, x^{\prime}, y\right) \rightarrow(t, x)$ by solving the equations

$$
\left\{\begin{array}{l}
t=t^{\prime} \\
x_{j}=x_{j}^{\prime}+\varphi(t, x) \bar{\varphi}(t, x) y_{j} \quad(\bar{\varphi} \text { denotes a complex conjugate of } \varphi)
\end{array}\right.
$$

and consider a hyperfunction

$$
f^{\prime}\left(t^{\prime}, x^{\prime}, y\right)=f(t, x) .
$$

Then, $f^{\prime}\left(t^{\prime}, x^{\prime}, y\right)$ is a hyperfunction on $\left(t^{\prime}, x^{\prime}, y\right)$-space and its singularity spectrum is contained in $\sqrt{-1} d t^{\prime} \infty$ over $t^{\prime}=x^{\prime}=y^{\prime}=0$. Moreover, $f^{\prime}\left(t^{\prime}, x^{\prime}, y\right)$ satisfies the holonomic system of the micro-differential equations with characteristic variety $V^{\prime}$. Hence we can apply the previous argument to $f^{\prime}\left(t^{\prime}, x^{\prime}, y\right)$. Note also that the image $Y^{\prime}$ of $V^{\prime}$ is

$$
\left\{\left(t^{\prime}, x^{\prime}, y\right) ;(t, x) \in Y\right\}=\left\{\left(t^{\prime}, x^{\prime}, y\right) ;\left(t^{\prime}, x^{\prime}\right) \in Y\right\} .
$$

because $\varphi(t, x)=0$ on $Y$.

Let $\widetilde{X^{\prime}-Y}(\widetilde{X-Y}$, resp. $)$ be the universal covering space of $\left\{\left(t^{\prime}, x^{\prime}, y\right) ;\left|t^{\prime}\right|<\delta,\left|x^{\prime}\right|\right.$ $\left.<\varepsilon,|y|<\varepsilon,\left(t^{\prime}, x^{\prime}\right) \notin Y\right\}(\{(t, x) ;|t|<\delta,|x|<\varepsilon,(t, x) \notin Y\}$, resp. $)$ and let $\varpi^{\prime}\left(\varpi^{\prime \prime}\right.$, resp. $)$ be the projection from $\overline{X^{\prime}-\bar{Y}}(\overline{X-Y}$, resp. $)$ to $\left(t^{\prime}, x^{\prime}, y\right)$-space $((t, x)$-space, resp.). Then we have a canonical map $\psi: \overline{X^{\prime}-Y} \rightarrow \overline{X-Y}$.

The previous argument shows that $\tilde{f^{\prime}}\left(t^{\prime}, x^{\prime}, y\right)=\tilde{f}(t, x)$ is analytically continued to a function defined on $\varpi^{\prime \prime-1}\left(\left\{\left(t^{\prime}, x^{\prime}, y\right) ; x^{\prime} \notin H\right\}\right)$. We shall show that $\tilde{f}(t, x)$ can be prolonged all over $\overline{X-Y}$. For any point $p$ in $\overline{X-Y}-\varpi^{-1}\left(\bar{Y} \cap F^{-1}(H)\right)(\bar{Y}$ is a complex conjugate of $Y)$, we can find $p^{\prime}$ in $\widetilde{X^{\prime}-Y}$ such that $\psi\left(p^{\prime}\right)=p$ and $\varpi^{\prime}\left(p^{\prime}\right)$ is outside $\left\{\left(t^{\prime}, x^{\prime}, y\right) ; x^{\prime} \in H\right\}$. In fact, for any $(t, x)$ outside $Y \cup \bar{Y}$, we can find $\left(t^{\prime}, x^{\prime}, y\right)$ over $(t, x)$ with $x^{\prime} \notin H$ for a suitable sufficiently small $y$, because $\varphi(t, x) \bar{\varphi}(t, x) \neq 0$. Therefore $\tilde{f}(t, x)$ can be prolonged to a function on $\overline{X-Y}-\varpi^{-1}\left(\bar{Y} \cap F^{-1}(H)\right)$. Since $\varpi^{-1}\left(\bar{Y} \cap F^{-1}(H)\right)$ is of complex codimension 2 , it is a removable singularity of $\tilde{f}$, and hence $\tilde{f}$ can be analytically continued all over $\widetilde{X-Y}$. Q.E.D.

Now we state our main result.

Theorem 1. Let $F_{1}(t, x)$ and $F_{2}(t, x)$ be microfunctions defined in a neighborhood of $(0$; $\sqrt{-1} d t \infty)$. Assume that $F_{j}(j=1,2)$ satisfies a holonomic system $\mathfrak{M}_{j}=\mathscr{E} / \mathscr{J}_{j}(j=1,2$, resp.) of micro-differential equations defined in a neighborhood of $(0 ; d t \infty)$ whose characteristic variety $V_{j}(j=1,2$, resp.) satisfies condition (8) in Lemma 1. Assume further that there exists a micro-differential oprator $Q\left(t, x, D_{t}, D_{x}\right)$ defined in a neighborhood of $(0 ; \sqrt{-1} d t \infty)$ which satisfies

$$
Q\left(t, x, D_{t}, D_{x}\right) F_{1}(t, x)=F_{2}(t, x) .
$$

Then there exists a multi-valued analytic function $\tilde{F}_{2}(t, x)\left(\tilde{F}_{1}(t, x)\right.$, resp. $)$ defined outside $Y=\pi\left(V_{1}\right)$ whose boundary value attains $F_{2}(t, x)\left(F_{1}(t, x)\right.$, resp. $)$, and they enjoy 
the following property for a sufficiently small neighborhood $\omega$ of the origin

$$
\text { If } \sum_{l=1}^{N} a_{l} T_{\gamma_{l}} \tilde{F}_{1}(t, x)\left(a_{l} \in \mathbb{C}\right) \text { is holomorphic in } \omega \text { for closed loops } \gamma_{l} \text { in } \omega-Y \text {, then }
$$
$\sum_{l=1}^{N} a_{l} T_{\gamma_{l}} \tilde{F}_{2}(t, x)$ is also holomorphic in $\omega$.

Proof. First note that the existence of $\tilde{F}_{1}$ and $\tilde{F}_{2}$ follows from Proposition 1.5.4 of Sato et al. [16], Chapter I combined with Lemma 3.

Condition (8) asserts that we can find generators $P_{j}\left(t, x, D_{t}, D_{x}\right)\left(j=1, \ldots, j_{0}\right)$ of $\mathscr{J}$ so that the common zero of their principal symbols intersects $\pi^{-1}(0)$ only at $\{d t \infty\}$. Therefore, by making use of Späth-type division theorem for micro-differential operators (Theorem 2.2.1 of Sato et al. [16], Chapter II), we can rewrite $Q\left(t, x, D_{t}\right.$, $\left.D_{x}\right)$ in the form

$$
\sum_{\substack{|\alpha| \leq m \\ \alpha j \leqq 0}} R_{\alpha}\left(t, x, D_{t}\right) D_{x}^{\alpha}+\sum_{j=1}^{j o} T_{j}\left(t, x, D_{t}, D_{x}\right) P_{j}\left(t, x, D_{t}, D_{x}\right)
$$

for micro-differential operators $R_{\alpha}$ and $T_{j}$. Since $P_{j}\left(j=1, \ldots, j_{0}\right)$ annihilates $F_{1}(t, x)$, we may assume from the first that $Q$ has the form $\sum_{\substack{|\alpha| \leqq m \\ \alpha, \leqq 0}} R_{\alpha}\left(t, x, D_{t}\right) D_{x}^{\alpha}$, and hence it
has the following form:

$$
\begin{aligned}
& Q\left(t, x, D_{t}, D_{x}\right)=Q_{d}\left(t, x, D_{t}, D_{x}\right)+Q_{m}\left(t, x, D_{t}, D_{x}\right) \\
& =Q_{d}\left(t, x, D_{t}, D_{x}\right)+\sum_{\substack{|\alpha| \leqq m \\
\alpha_{j} \leqq 0}} Q_{\alpha}\left(t, x, D_{t}\right) D_{x}^{\alpha},
\end{aligned}
$$

where $Q_{d}\left(t, x, D_{t}, D_{x}\right)$ is a linear differential operator and $Q_{\alpha}\left(t, x, D_{t}\right)$ is a microdifferential operator of order less than zero which does not contain $D_{x}$.

Before discussing the procedure of analytic continuation, we note that Lemma 2 claims that $\gamma_{l}(l=1, \ldots, N)$ can be chosen so that it defines an element in $\pi_{1}\left(F^{-1}(x) \cap(\omega-Y)\right)$ for sufficiently small $\omega$ with $x \notin H$ (see Lemma 2 for the

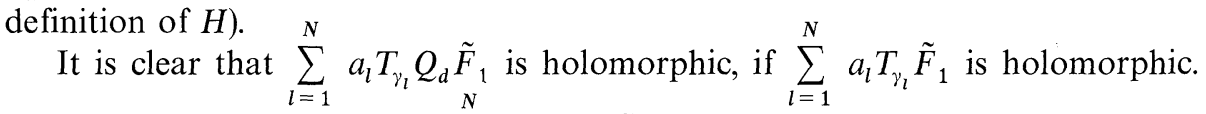
Hence it suffices to show that $\sum_{l=1}^{N} a_{l} T_{\gamma_{l}} Q_{m} \tilde{F}_{1}$ is holomorphic. Since $Q_{\alpha}\left(t, x, D_{t}\right)$ is a micro-differential operator of order less than zero, we can find holomorphic function $q_{\alpha}\left(t, t^{\prime}, x\right)$ defined near $\left(t, t^{\prime}, x\right)=(0,0,0)$ so that $Q_{\alpha}\left(t, x, D_{t}\right) D_{x}^{\alpha} F_{1}(t, x)$ is equal to a boundary value of holomorphic function defined by

$$
\int_{c}^{t} q_{\alpha}\left(t, t^{\prime}, x\right) D_{x}^{\alpha} \tilde{F}_{1}\left(t^{\prime}, x\right) d t^{\prime}
$$

at $(0, \sqrt{-1} d t \infty)$ for sufficiently small $c$ with $\operatorname{Im} c>0$. (Kashiwara and Kawai [2], §3.) Note that $\int_{c}^{t} q_{\alpha}\left(t, t^{\prime}, x\right) D_{x}^{\alpha} \tilde{F}_{1}\left(t^{\prime}, x\right) d t^{\prime}$ is also a multi-valued function on $X-Y$, and hence the singularity spectrum at $(t, x)=(0,0)$ of this boundary value is contained in $\sqrt{-1} d t \infty$. Therefore, $\tilde{F}_{2}(t, x)-\sum_{\alpha} \int_{c}^{t} q_{\alpha}\left(t, t^{\prime}, x\right) D_{x}^{\alpha} \tilde{F}_{1}\left(t^{\prime}, x\right) d t^{\prime}$ is holomorphic in a 
neighborhood of $(t, x)=(0,0)$, because its boundary value has no singularity spectrum.

Making use of this concrete expression of $Q_{\alpha}\left(t, x, D_{t}\right) D_{x}^{\alpha} F_{1}(t, x)$, we shall show that

$$
\begin{aligned}
& F(t, x)=\sum_{l=1}^{N} a_{l} T_{\gamma_{l}} \sum_{\alpha} \int_{c}^{t} q_{\alpha}\left(t, t^{\prime}, x\right) D_{x}^{\alpha} \tilde{F}_{1}\left(t^{\prime}, x\right) d t^{\prime} \\
& -\sum_{l=1}^{N} a_{l} \sum_{\alpha} \int_{c}^{t} q_{\alpha}\left(t, t^{\prime}, x\right) D_{x}^{\alpha} T_{\gamma_{l}} \tilde{F}_{1}\left(t^{\prime}, x\right) d t^{\prime}
\end{aligned}
$$

is holomorphic in a neighborhood of the origin.

If this is the case, then the proof will be complete. In fact, the first term is equal to $\sum_{l=1}^{N} a_{l} T_{\gamma_{l}} \tilde{F}_{2}$ modulo holomorphic function and $\sum_{l=1}^{N} a_{l} \sum_{\alpha} \int_{c}^{t} q_{\alpha}\left(t, t^{\prime}, x\right) D_{x}^{\alpha} T_{\gamma_{l}} \tilde{F}_{1}\left(t^{\prime}, x\right) d t^{\prime}$ is holomorphic in a neighborhood of the origin, because

$$
\sum_{l=1}^{N} a_{l} T_{\gamma_{l}} \tilde{F}_{1}\left(t^{\prime}, x\right)
$$

is holomorphic in a neighborhood of the origin by the assumption.

Now we shall calculate $F(t, x)$. First $T_{\gamma_{l}} \int_{c}^{t} q_{\alpha}\left(t, t^{\prime}, x\right) D_{x}^{\alpha} \tilde{F}_{1}\left(t^{\prime}, x\right) d t^{\prime}$ is an integral of $q_{\alpha}\left(t, t^{\prime}, x\right) D_{x}^{\alpha} \tilde{F}_{1}\left(t^{\prime}, x\right) d t^{\prime}$ along the path in Figure 1a. There $\gamma_{l}(t)$ signifies the point over $t$ on the sheet relevalent to $\gamma_{l}$. Secondly $\int_{c}^{t} q_{\alpha}\left(t, t^{\prime}, x\right) D_{x}^{\alpha} T_{\gamma_{l}} \tilde{F}_{1}\left(t^{\prime}, x\right) d t^{\prime}$ is an
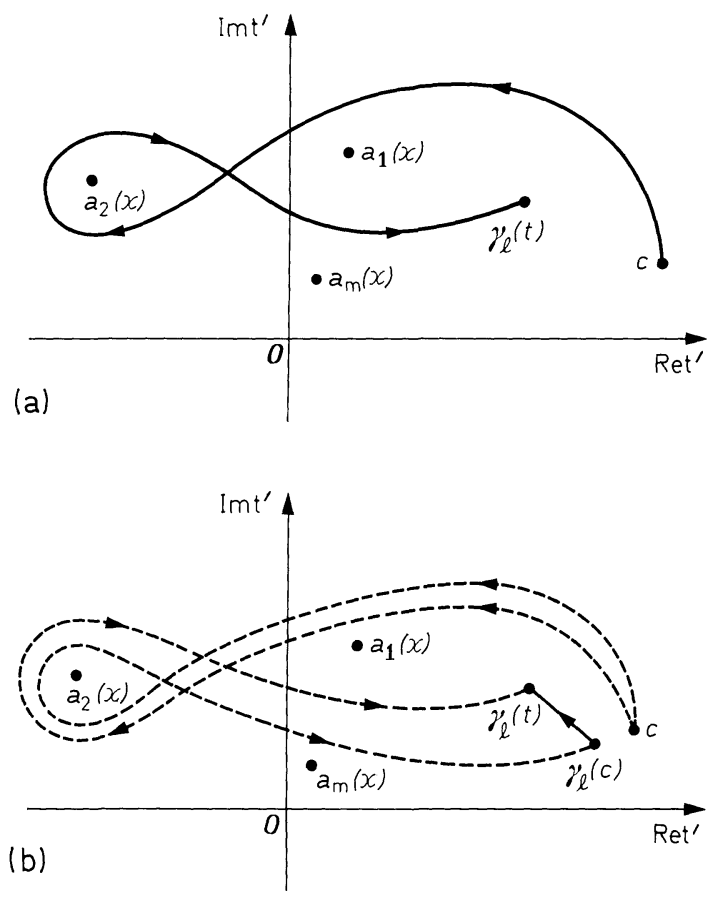

Fig. 1 
integral of $q_{\alpha}\left(t, t^{\prime}, x\right) D_{x}^{\alpha} \tilde{F}_{1}\left(t^{\prime}, x\right) d t^{\prime}$ along the path from $\gamma_{l}(c)$ to $\gamma_{l}(t)$. Therefore, as seen in Figure $1 b$

$$
\begin{aligned}
& T_{\gamma_{l}} \int_{c}^{t} q_{\alpha}\left(t, t^{\prime}, x\right) D_{x}^{\alpha} \tilde{F}_{1}\left(t^{\prime}, x\right)-\int_{c}^{t} q_{\alpha}\left(t, t^{\prime}, x\right) D_{x}^{\alpha} T_{\gamma_{l}} \tilde{F}_{1}\left(t^{\prime}, x\right) d t^{\prime} \\
& =\int_{c}^{\gamma_{l}(t)} q_{\alpha}\left(t, t^{\prime}, x\right) D_{x}^{\alpha} \tilde{F}_{1}\left(t^{\prime}, x\right) d t^{\prime} \\
& \quad-\int_{\gamma_{l}(c)}^{\gamma_{l}(t)} q_{\alpha}\left(t, t^{\prime}, x\right) D_{x}^{\alpha} \tilde{F}_{1}\left(t^{\prime}, x\right) d t^{\prime}
\end{aligned}
$$

is an integral of $q_{\alpha}\left(t, t^{\prime}, x\right) D_{x}^{\alpha} \tilde{F}_{1}\left(t^{\prime}, x\right) d t^{\prime}$ along the path from $t^{\prime}=c$ to $t^{\prime}=\gamma_{l}(c)$.

Therefore, $F(t, x)$ is analytic as long as this contour is not trapped between the singularities of the integrand, i.e., as long as $x \notin H$. This means that $F(t, x)$ is analytic except possibly at $x \in H$.

On the other hand,

$$
\varphi(t, x)=\sum_{\alpha} \int_{c}^{t} q_{\alpha}\left(t, t^{\prime}, x\right) D_{x}^{\alpha} \tilde{F}_{1}\left(t^{\prime}, x\right) d t^{\prime}-\tilde{F}_{2}(t, x)
$$

is a holomorphic function defined near the origin as seen before. Therefore $\sum_{l=1}^{N} a_{l} T_{\gamma_{l}}$ $\sum_{\alpha} \int_{c}^{t} q_{\alpha}\left(t, t^{\prime}, x\right) D_{x}^{\alpha} \tilde{F}_{1}\left(t^{\prime}, x\right) d t^{\prime}-\left(\sum_{l=1}^{N} a_{l}\right) \varphi(t, x)$ should satisfy $\mathfrak{M}_{2}$, hence it must be analytic outside $\pi\left(V_{2}\right)$. Since $\varphi$ is holomorphic in a neighborhood of the origin, and that the second term in (36) is holomorphic, $F(t, x)$ must be holomorphic in a neighborhood of the origin.

This completes the proof of the theorem.

\section{§3. An Application to Feynman Integrals}

In this section we show how the results in $\$ 2$ can be applied to analyze the "hierarchy" of Feynman integrals. In order to avoid technical difficulties related to renormalization procedure, we restrict our investigation to the simplest case in this paper.

In this section we use the same notations as in Nakanishi [8], Speer [17], Kashiwara and Kawai [3, 4], and Kawai and Stapp [5, 6] and do not repeat their definitions.

In the sequel we exclusively consider Feynman integral $F_{D}(p)$ associated with a Feynman diagram $D$ which is external in the sense that at least one external line is attached to each vertex $j$ of $D$. Then we may assume without lose of generality that exactly one external line is coming into each vertex, since we discuss off-shell amplitudes in this article.

We also assume that all the relevalent particles are massive and spinless.

We first consider generalized Feynman integral $F_{D}(p ; \lambda)$ formally defined below (Speer [17])

$$
F_{D}(p ; \lambda)=\int_{\mathbb{R}^{4 N}} \frac{\prod_{j=1}^{N} \delta^{4}\left(p_{j}+\sum_{l=1}^{N}[j: l] k_{l}\right)}{\prod_{l=1}^{N}\left(k_{l}^{2}-m_{l}^{2}+i 0\right)^{\lambda_{l}}} \prod_{l=1}^{N} d^{4} k_{l}
$$


We denote generalized Feynman amplitude by $f_{D}(p ; \lambda)$, that is, $f_{D}(p ; \lambda)$ is defined on $M=\left\{p \in \mathbb{R}^{4 n} ; \sum_{j=1}^{n} p_{j}=0\right\}$ and satisfies $F_{D}(p ; \lambda)=\delta^{4}\left(\sum_{j=1}^{n} p_{j}\right) f_{D}(p ; \lambda)$. A complexification $X$ of $M$ is defined by $\left\{p \in \mathbb{C}^{4 n} ; \sum_{j=1}^{n} p_{j}=0\right\}$.

It is known (Kashiwara and Kawai [4]) that $F_{D}(p, \lambda)$ can be considered as an integral over $\left(P\left(\mathbb{R}^{4}\right)\right)^{N}$, and that it satisfies a holonomic system of linear differential equations for generic $\lambda$. Furthermore, the characteristic variety of $\mathfrak{M}_{\lambda}$ is contained in the extended Landau variety $\tilde{\mathscr{L}}(D)$ defined below (Kashiwara and Kawai [4], Theorem 2).

$\tilde{\mathscr{L}}(D)=\left\{(p, u) \in T^{*}\left(\mathbb{C}^{4 n}\right)\right)$; there exist a sequence of scalars $c_{l}^{(m)}$ and $\alpha_{l}^{(m)}(l$ $=1, \ldots, N)$ and four-vectors $p_{j}^{(m)}, u_{j}^{(m)}(j=1, \ldots, n)$ and $k_{l}^{(m)}(l=1, \ldots, N)$ which satisfy the following relation (38)\}

$$
\begin{cases}p_{j}^{(m)} \rightarrow p_{j} & j=1, \ldots, n \\ u_{j}^{(m)} \rightarrow u_{j} & j=1, \ldots, n \\ p_{j}^{(m)}+\sum_{l=1}^{N}[j: l] k_{l}^{(m)}=0 & j=1, \ldots, n \\ \left(\sum_{j=1}^{n}[j: l] u_{j}^{(m)}+\alpha_{l}^{(m)} k_{l}^{(m)}\right) / c_{l}^{(m)} \rightarrow 0 & l=1, \ldots, N \\ \alpha_{l}^{(m)}\left(k_{l}^{(m)^{2}}-m_{l}^{2}\right) \rightarrow 0 & l=1, \ldots, N \\ c_{l}^{(m)} \text { is bounded } & l=1, \ldots, N \\ c_{l}^{(m)} k_{l}^{(m)} \text { is bounded } & l=1, \ldots, N \\ \left(c_{l}^{(m)}, c_{l}^{(m)} k_{l}^{(m)}\right) \nrightarrow 0 & l=1, \ldots, N .\end{cases}
$$

Denote by $\tilde{\mathscr{L}}(D)_{\infty}$ the subvariety of $\tilde{\mathscr{L}}(D)$ defined by the same equations as (38) with the additional condition

(39) $c_{l}^{(m)} \rightarrow 0$ for some $l$.

Note that $\tilde{\mathscr{L}}(D)_{\infty}$ corresponds to the so-called (mixed) second-type singularities. Note also that the Equation (38) reduces to the following Equation (40) [((ordinary)Landau(-Nakanishi)equations] if $(p, u) \in \tilde{\mathscr{L}}(D)-\tilde{\mathscr{L}}(D)_{\infty}$. Here we have used the externality condition on the diagram $D$.

$$
\begin{aligned}
\mathscr{L}(D)=\{ & (p, u) \in P^{*}\left(\mathbb{C}^{4 n}\right) ; p_{j}+\sum_{l=1}^{N}[j: l] k_{l}=0 \quad(j=1, \ldots, n), \\
& \sum_{j=1}^{n}[j: l] u_{j}+\alpha_{l} k_{l}=0 \quad(l=1, \ldots, N), \\
& \left.\alpha_{l}\left(k_{l}^{2}-m_{l}^{2}\right)=0 \quad(l=1, \ldots, N) \quad \text { and } \quad \alpha_{l_{0}} \neq 0 \quad \text { for some } l_{0}\right\} .
\end{aligned}
$$

For the sake of simplicity of notations, we define $\mathscr{L}_{1}(D)$ by $\left\{(p, u) \in P^{*}\left(\mathbb{C}^{4 n}\right) ; p_{j}\right.$ $+\sum_{l=1}^{N}[j: l] k_{l}=0(j=1, \ldots, n), \sum_{j=1}^{n}[j: l] u_{j}+\alpha_{l} k_{l}=0(l=1, \ldots, N), \alpha_{1}\left(k_{1}^{2}-m_{1}^{2}\right)=0, k_{l}^{2}$ $-m_{l}^{2}=0$ and $\left.\alpha_{l} \neq 0(l=2, \ldots, N)\right\}$. 
We similarly define $\mathscr{L}_{0}(D)$ by $\left\{(p, u) \in P^{*}\left(\mathbb{C}^{4 n}\right) ; p_{j}+\sum_{l=1}^{N}[j: l] k_{l}=0(j=1, \ldots, n)\right.$,
$\sum_{j=1}^{n}[j: l] u_{j}+\alpha_{l} k_{l}=0(l=1, \ldots, N), k_{l}^{2}-m_{l}^{2}=0$ and $\left.\alpha_{l} \neq 0(l=1, \ldots, N)\right\}$.

We denote by $D^{\prime}$ the diagram obtained by contracting out exactly one internal line of $D$, say the first internal line. The Feynman integral $F_{D^{\prime}}(p ; \lambda)$ is given by

$$
\int \frac{\prod_{[j: 1]=0} \delta^{4}\left(p_{j}+\sum_{l=2}^{N}[j: l] k_{l}\right) \delta^{4}\left(p_{j^{+}}+p_{j^{-}}+\sum_{l=2}^{N}\left[j^{+}: l\right] k_{l}+\sum_{l=2}^{N}\left[j^{-}: l\right] k_{l}\right)}{\prod_{l=2}^{N}\left(k_{l}^{2}-m_{l}^{2}+i 0\right)^{\lambda_{l}}} \prod_{l=2}^{N} d^{4} k_{l} .
$$

Here $j^{ \pm}$denotes the (unique) number that satisfies $\left[j^{ \pm}: 1\right]= \pm 1$.

We define $\mathscr{L}_{0}\left(D^{\prime}\right)$ as follows: $\mathscr{L}_{0}\left(D^{\prime}\right)=\left\{(p, u) \in P^{*}\left(\mathbb{C}^{4 n}\right) ; p_{j}+\sum_{l=2}^{N}[j: l] k_{l}=0\right.$ for $j$ with $[j: 1]=0, p_{j^{+}}+p_{j^{-}}+\sum_{l=2}^{N}\left[j^{+}: l\right] k_{l}+\sum_{l=2}^{N}\left[j^{-}: l\right] k_{l}=0, k_{l}^{2}-m_{l}^{2}=0$ and $\alpha_{l} \neq 0$ $\left.(l=2, \ldots, N), \sum_{j=1}^{n}[j: l] u_{j}+\alpha_{l} k_{l}=0(l=1, \ldots, N), \alpha_{1}=0\right\}$.

Using these notations our theorem is stated as follows:

Theorem 2. Let $p_{0} \in \mathbb{R}^{4 n}$ belong to $\pi\left(\mathscr{L}_{0}(D) \cap \mathscr{L}_{0}\left(D^{\prime}\right)\right)$. Assume further that there exists a complex neighborhood $\omega_{0}$ of $p_{0}$ which satisfies the following:

$$
\pi^{-1}\left(\omega_{0}\right) \cap \tilde{\mathscr{L}}(D)_{\infty}=\left\{(p, u) ; p \in \omega_{0}, \sum_{j=1}^{n} p_{j}=0 \text { and } u_{j}=a \text { for some } a \in \mathbb{C}^{4}\right\} .
$$

(42) If $(p, u)$ belongs to $\pi^{-1}\left(\omega_{0}\right) \cap \mathscr{L}(D)$, then corresponding $(\alpha, k)$ should satisfy $(40)$.

(43) $\pi\left(\mathscr{L}_{1}(D)\right) \cap \omega_{0}$ has the form $S_{1} \cup S_{2}$ with non-singular hypersurface $S_{1}$ and $S_{2}$ in $X$ which touch mutually tangentially along $S_{1} \cap S_{2}$, which is non-singular and codimension 1 in $S_{1}$ and $S_{2}$.

Then there exist a neighborhood $\omega$ of $p_{0}$ and a multi-valued analytic function $\tilde{f}_{D}(p)$ $\left(\tilde{f}_{D^{\prime}}(p)\right.$, resp.) defined on $\omega \cap X-\pi\left(\mathscr{L}_{1}(D)\right)\left(\omega \cap X-\pi\left(\mathscr{L}_{0}\left(D^{\prime}\right)\right)\right.$, resp.) whose boundary value attains the Feynman amplitude $f_{D}(p)\left(f_{D^{\prime}}(p)\right.$, resp. $)$, and they enjoy the following property:

If $\sum_{l=1}^{m} a_{l} T_{\gamma_{l}} \tilde{f}_{D}(p)\left(a_{l} \in \mathbb{C}\right)$ is holomorphic in $\omega \cap X$ for closed loops $\gamma_{l}$ in $\omega \cap X-\pi\left(\mathscr{L}_{1}(D)\right)$, then $\sum_{l=1}^{m} a_{l} T_{\gamma_{l}} \tilde{f}_{D^{\prime}}(p)$ is also holomorphic in $\omega \cap X$.

Proof. It suffices to show that conditions required in Theorem 1 are fulfilled in this case.

In view of conditions (41) and (42), Theorem 2.1.1 of Sato et al. [16] entails that S.S. $f_{D}(p ; \lambda)$ is confined to $\mathscr{L}(D) \cap \sqrt{-1} S^{*} M$ in $\pi^{-1}\left(\omega_{0}\right)$. Note that $\mathscr{L}(D)$ naturally defines a subvariety of $P^{*} X$ under the usual convention that $(p, u)=\left(p^{\prime}, u^{\prime}\right)$ if and only if $p=p^{\prime}$ and $u_{j}-u_{j}^{\prime}=a(j=1, \ldots, n)$ for some $a \in \mathbb{C}^{4}$. 
Furthermore, taking account of the $+i 0$ character of the propagator, one can easily verify that S.S. $f_{D}(p, \lambda)$ is really contained in the positive- $\alpha$ Landau(-Nakanishi) variety, i.e., S.S. $f_{D}(p, \lambda) \subset\left\{(p, \sqrt{-1} u) \in \sqrt{-1} S^{*} M ; p_{j}+\sum_{l=1}^{N}[j: l] k_{l}=0 \quad(j=1, \ldots, n)\right.$, $\left.\sum_{j=1}^{n}[j: l] u_{j}+\alpha_{l} k_{l}=0(l=1, \ldots, N), \alpha_{l}\left(k_{l}^{2}-m_{l}^{2}\right)=0(l=1, \ldots, N), \alpha_{l} \geqq 0\right\}$ (cf. Sato et al. [16], Chapter I, §2). Moreover $F_{D}(p, \lambda)$ considered as a microfunction depends holomorphically on $\lambda \in \mathbb{C}^{N}$ in $\pi^{-1}\left(\omega_{0}\right)$ by condition (41). Therefore $F_{D}(p ; 1, \ldots, 1)$ is well-defined as a microfunction and is equal to

$$
F_{D}(p)=\int \frac{\prod_{j=1}^{n} \delta^{4}\left(p_{j}+\sum_{l=1}^{N}[j: l] k_{l}\right)}{\prod_{l=1}^{N}\left(k_{l}^{2}-m_{l}^{2}+i 0\right)} \prod_{l=1}^{N} d^{4} k_{l} .
$$

Hence $f_{D}(p ; 1, \ldots, 1)=f_{D}(p)$. Then one of our results on the relationship between $F_{D}(p)$ and $F_{D^{\prime}}(p)$ claims that there exists a micro-differential operator $Q\left(p, D_{p}\right)$ defined in a neighborhood of $\left(p_{0}, u_{0}\right) \in \overline{\mathscr{L}_{0}(D)} \cap \mathscr{L}_{0}\left(D^{\prime}\right)$ such that

$$
Q\left(p, D_{p}\right) f_{D}(p)=f_{D^{\prime}}(p)
$$

holds there (see Sato [15], p. 22).

Furthermore in a neighborhood of $\left(p_{0}, u_{0}\right) \in \overline{\mathscr{L}_{0}(D)} \cap \mathscr{L}_{0}\left(D^{\prime}\right) f_{D}(p)$ satisfies a holonomic system of micro-differential equations whose characteristic variety is confined to $\mathscr{L}_{1}(D)$. This fact follows from conditions (41) and (42) combined with Theorem 2 of Kashiwara and Kawai [4]. One can also verify this results under conditions (41) and (42) by the immediate application of Theorems 3.5.3 and 3.5.5 to $f_{D}(p)$ (cf. Sato $\left.[15]\right)^{4}$.

Thus we find that all the conditions required by Theorem 1 are fulfilled in our case, if we choose a local coordinate system $(t, x)$ on $M$ in a neighborhood of $p_{0}$ so that $S_{1}$ has the form $\{t=0\}$ and that S.S. $f_{D}(p) \cap \pi^{-1}(0)=\{\sqrt{-1} d t \infty\}$. Condition (43) guarantees that such a choice of a local coordinate system is possible. Note that $\mathscr{L}_{1}(D) \cap \pi^{-1}\left(\omega_{0}\right)$ is a conormal set of $S_{1} \cup S_{2}$ under condition (43). Therefore we find the required relationship between $f_{D}(p)$ and $f_{D^{\prime}}(p)$. Q.E.D.

Remark 1 . Though we have restricted ourselves to the case where exactly one simple internal line is contracted out, we can equally deal with the case where multiple lines are contracted out. However, since the proof of the existence of micro-differential operator $Q\left(p, D_{p}\right)$ in this case requires a detailed argument on renormalization, we will discuss this case in a separate paper.

Remark 2. Theorems 1 and 2 might be regarded as a micro-local version of the celebrated "hierarchical principle" in perturbation theory (see Landshoff et al. [7]).

Acknowledgments. We would like to express our heartiest thanks to Professor M. Sato, Professor H. Stapp, and Professor T. Regge for very stimulating and interesting discussions with them. We would also like to express our heartiest thanks to Professor T. Kato for kindly inviting one of us (M.K.) to Berkeley and making it possible for us to collaborate on the topics discussed in this paper.

4 This is because there is no " $u=0$ point" for off-shell amplitude except possibly where the points at infinity with respect to $k$ are relevalent, if $D$ is supposed to be external. See Kawai and Stapp $[5,6]$ for this point 


\section{References}

1. Barucchi,G., Ponzano, G. : J. Math. Phys. 14, 390-401 (1973)

2. Kashiwara, M., Kawai, T.: J. Math. Soc. Japan 27, 359-404 (1975)

3. Kashiwara,M., Kawai, T.: Proc. Japan Acad. 52, 161-164 (1976)

4. Kashiwara,M., Kawai,T.: Holonomic systems of linear differential equations and Feynman integrals. To appear in Proc. Oji Seminar (Publ. RIMS 12 supplement)

5. Kawai,T., Stapp,H.P.: Micro-local study of the $S$-matrix singularity structure. In: Lecture note in physics, No. 39, pp. 38-48. Berlin-Heidelberg-New York: Springer 1975

6. Kawai,T., Stapp,H.P. : Discontinuity formula and Sato's conjecture. To appear in Proc. Oji Seminar (Publ. RIMS, 12 supplement)

7. Landshoff, P. V., Olive, D. I., Polkinghorne,J.C.: Nuovo Cimento 43, 444-453 (1966)

8. Nakanishi, N.: Graph theory and Feynman integrals. New York-London-Paris: Gordon and Breach 1971

9. Pham,F.: Ann. Inst. H.Poincaré 6 A, 89-204 (1967)

10. Ponzano, G., Regge,T., Speer,E.R., Westwater, M.J.: Commun. math. Phys. 15, $83-132$ (1969)

11. Ponzano,G., Regge,T., Speer,E. R., Westwater,M.J.: Commun. math. Phys. 18, 1-64 (1970)

12. Regge, T.: Algebraic topology methods in the theory of Feynman relativistic amplitudes. In : Report of Battele rencontres, pp. 433-458. New York: Benjamin 1968

13. Regge, T.: Feynman integrals. In: Actes Congrès intern. Math. (Nice), Tome 3, pp. 9 -14. Paris : Gauthier-Villars 1970

14. Regge, T., Speer, E. R., Westwater, M.J.: Fortschr. Phys. 20, 365-420 (1972)

15. Sato, M.: Recent development in hyperfunction theory and its application to physics. In: Lecture notes in physics, No. 39, pp. 13-29. Berlin-Heidelberg-New York: Springer 1975

16. Sato,M., Kawai,T., Kashiwara, M.: Microfunctions and pseudo-differential equations. In: Lecture notes in math., No. 287, pp. 265-529. Berlin-Heidelberg-New York: Springer 1973

17. Speer,E. R.: Generalized Feynman amplitudes. Princeton: Princeton University Press 1969

18. Speer,E. R., Westwater,M.F.: Ann. Inst. H.Poincaré 14A, 1-55 (1971)

Communicated by H. Araki

Received August 2, 1976; in revised form October 12, 1976 